journal club

\title{
Eine echte Alternative zu Carbamazepin
}

Fragestellung: Lacosamid (LCM) ist ein bereits langjährig etabliertes antikonvulsives Medikament bei fokalen Epilepsien. Ist es in Monotherapie genauso effektiv und darüber hinaus vergleichbar verträglich wie das gut etablierte und bekanntermaßen wirkstarke Carbamazepin (CBZ)?

Hintergrund: LCM ist ein gut etabliertes Antikonvulsivum der dritten Generation und seit 2008 in Kombinationstherapie bei fokalen Epilepsien mit und ohne sekundäre Generalisation zugelassen. Der Wirkmechanismus funktioniert ähnlich dem des CBZ auf dem Boden der Hemmung spannungsabhängiger Natriumkanäle, ohne jedoch dabei die schnelle Inaktivierung zu beeinflussen. Durch die langsame Inaktivierung der Natriumkanäle stabilisiert es das neuronale Ruhemembranpotenzial, sodass es nicht zu weiteren überschüssigen Aktionspotenzialen kommen kann. Auch scheint es einen Effekt auf die CRMP-2-Rezeptoren zu haben. Bezüglich der Wirksamkeit muss es sich in dieser Studie gegen retardiertes CBZ, dem Wirkklassiker bei fokalen Epilepsien bewähren. Auch die Verträglichkeit steht im Fokus.

Baulac M, Rosenow F, Toledo $M$ et al. Efficacy, safety, and tolerability of lacosamide monotherapy versus controlled-release carbamazepine in patients with newly diagnosed epilepsy: a phase 3, randomised, doubleblind, non-inferiority trial. Lancet Neurol 2017; 16: $43-54$
Patienten und Methodik: In die doppelblinde, randomisierte und multizentrische Phase-III-Studie wurden 888 Patienten, die älter als 16 Jahre waren, mit einer neu diagnostizierten fokalen Epilepsie eingeschlossen. Die Randomisierung erfolgte computer- gesteuert im Verhältnis 1: 1 entweder auf LCM oder retardiertes CBZ, jeweils in Monotherapie und einer auf zwei Einzelgaben verteilten Tagesdosis. Die Einstiegsdosis lag für CBZ bei $200 \mathrm{mg}$, für LCM bei $100 \mathrm{mg} /$ Tag. Über die folgenden zwei Wochen der Eindosierungsphase wurde CBZ auf $400 \mathrm{mg}$ und LCM auf $200 \mathrm{mg} /$ Tag eingestellt. Während des dann sechsmonatigen Assessments wurde die Dosis nur erhöht, wenn es zu neuen Anfällen kam. Die maximal verordnete Dosis lag bei $1.200 \mathrm{mg}$ CBZ oder $600 \mathrm{mg}$ LCM/Tag. In der sechsmonatigen Erhaltungsphase wurden keinerlei Medikamentenveränderungen mehr vorgenommen. Entscheidendes Ziel war Anfallsfreiheit während der an die Dosisfindungsphase anschließenden sechsmonatigen Erhaltungsphase und letztlich über den gesamten Studienzeitraum von zwölf Monaten. Wer anfallsfrei blieb, hatte nachfolgend die Option, in eine Studienverlängerung einzutreten.

Ergebnisse: 444 Patienten erhielten LCM, 442 CBZ. Anfallsfrei über mehr als sechs Monate blieben 74\% unter LCM und $70 \%$ unter CBZ-Monotherapie. Jeweils $60 \%$ der Teilnehmer in beiden Behandlungsarmen beendeten den kompletten Studienzeitraum. Hauptgründe, die Behandlung abzubrechen, waren in der LCMGruppe Schwindel und Hautausschlag, bei der CBZ-Gruppe Hautausschlag, erhöhte Leberwerte und störende Schläfrigkeit. Dabei wurden $7 \%$ der Nebenwirkungen bei LCM und $10 \%$ bei CBZ als schwerwiegendes unerwünschtes Ereignis klassifiziert.

Schlussfolgerung: Lacosamid ist retardiertem Carbamazepin in Monotherapie bei neu diagnostizierten fokalen Epilepsien nicht unterlegen und kann gleichermaßen first-line eingesetzt werden.

\section{- Kommentar von Vivien Homberg, Bad Berka}

\section{Klasse-1-Evidenz zum primären Einsatz als Monotherapie}

Diese interessante Studie ist immerhin schon die vierte Headto-head-Monotherapiestudie, die seitens der EMA und der ILAE in ähnlichem Design in Auftrag gegeben wurde und eine relevante Alltagsfrage klärt. Aufgrund des guten Wirksamkeits- und Verträglichkeitsprofils von LCM hat das Medikament nach dieser aktuell erhobenen Datenlage absolut Klasse-1-Evidenz zum primären Einsatz als Monotherapie bei neudiagnostizierten fokalen Epilepsien. Dies scheint sogar für das träge Zulassungsverfahren in Deutschland überzeugend gewesen zu sein, denn seit Januar 2017 ist LCM in Monotherapie auch bei uns verschreibbar. In Anbetracht der geringen Interaktion von Lacosamid im Gegensatz zu Carbamazepin und der letztlich guten Verträglichkeit, wäre es auch zu schade gewesen, es entsprechender Patientenklientel first-line vorenthalten zu müssen. Denn gerade ein gut funktionierendes, das heißt wirksames und verträgliches erstes antikonvulsives Medikament, das oftmals über Jahre, wenn nicht sogar lebenslang eingenommen werden muss, stärkt die Compliance und eine positive Arzt-Patientenbindung. Einen interessanten Neben- aspekt hat das aktuelle Studiendesign: die Entscheidung zu einer höheren LCM-Dosis von bis zu 600 mg/Tag in Monotherapie. Die Kombinationsstudien hatten bei > 400 mg LCM-Tagesdosis eine teils deutlich erhöhte Nebenwirkungsrate gezeigt, sodass die Zulassungsgrenze auf $400 \mathrm{mg} /$ Tag festgelegt wurde. Dies scheint in Monotherapie nicht mehr das Problem zu sein, was glücklicherweise in der aktuellen Zulassung auch entsprechend berücksichtigt wurde.

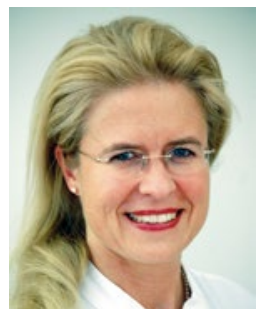

Dr. med. Vivien Homberg, Bad Berka

Chefärztin der Klinik für Neurologie, Zentralklinikum Bad Berka

E-Mail: Vivien.Homberg@zentralklinik.de 